\title{
Manganese-Enhanced MRI of Hypoxic-Ischemic Brain Injuries using Mn-DPDP
}

\author{
Jian Yang and Ed X. Wu
}

Abstract: In this study, Mn-dipyridoxaldiphosphate (MnDPDP), a clinically approved manganese contrast agent for hepatic and pancreatic imaging, was demonstrated for the first time for manganese-enhanced MRI (MEMRI) in brains of normal young rats $(n=4)$ and rats with hypoxic-ischemic (H-I) insult at postnatal day 7 $(n=8)$. After a single intraperitoneal injection of low dosage with $0.1 \mu \mathrm{mol} / \mathrm{g}$ in postnatal 14 days, 2D T1-weighted image (T1WIs), T1 maps, T2-weighted images (T2WIs) and T2 maps were acquired at 7 Tesla 1 day before, 1 day and 7 days after MnDPDP injection. The image contrast changes induced by MnDPDP appeared as the hyperintensity in T1WIs and the hypointensity in T2WIs. T1and T2 values decreased in the regions of Mn enhancement. Such enhancement presented as a delayed pattern that was more pronounced in 7 day after MnDPDP injection, suggesting the sustained Mn accumulation due to MnDPDP. Moreover, the MnDPDP enhancement in $\mathrm{H}-\mathrm{I}$ brains was more pronounced in the lesion sites and was easily detectable in T1WI, T1 map, T2WI and T2 map. The results demonstrated here support the possibility of using MnDPDP as a 'slow release' $\mathrm{Mn}^{2+}$ for clinical diagnosis of various neuropathologies.

\section{INTRODUCTION}

$\mathrm{D}$ IVALENT manganese ion $\left(\mathrm{Mn}^{2+}\right)$ as an analogue for calcium ion $\left(\mathrm{Ca}^{2+}\right)$ has been used as a MRI contrast agent to detect neuronal activation[1,2], neural architecture[3, 4], and neuronal connections [5, 6]. It is also essential to the development and function of the brain in physiological and pathological states [7-10]. Mn metal is bound to glutamine synthetase (GS) which is a glial specific enzyme for regulating the extracellular glutamate and ammonia. It can also reduce glutamate excitotoxicity [11-13] and to the mitochondrial Mn-superoxide dismutase (Mn-SOD) enzyme which acts against cellular oxidative stress [11, 14, 15]. Therefore, a previous study found that endogenous $\mathrm{Mn}$ in the form of Mn-SOD and GS were accumulated in delayed

This work was supported in part by the Hong Kong Research Grant Council (GRF HKU 7793/08M), The University of Hong Kong CRCG grant and The Shannxi Province Nature Science Grant. Jian Yang is with Medical Imaging Center of the First Affiliated Hospital, School of Medicine of Xi'an Jiaotong University Xi'an, Shannxi Province, China (e-mail: yj1118@mail.xjtu.edu.cn)

Ed X. Wu is with the Laboratory of Biomedical Imaging and SignalProcessing and the Department of Electrical and Electronic Engineering, The University of Hong Kong, Pokfulam, Hong Kong (corresponding author to provide phone: (852) 2819-9713; fax: (852) 2819-9711; e-mail: ewu@eee.hku.hk). neurodegenerated regions and could be detected by MRI in adult rat and human after experiencing stroke [11]. Recently, our studies demonstrated that exogenous Mn could provide enhanced MRI detection of oxidative stress and gliosis in neonatal rats with hypoxia-ischemia (H-I) and adult rats with focal photothrombotic cortical injury and transient middle cerebral artery occlusion [16-18]. Especially the upregulation of MnSOD and GS activities in gray matter with delayed neurodegeneration were observed in neonatal rats with mild $\mathrm{H}-\mathrm{I}$ injury, leading to Mn-enhanced MRI (MEMRI) detection of lesions undetectable by other MR modalities[18]. It suggests the potential usage of MEMRI by $\mathrm{Mn}^{2+}$ agent for clinical diagnosis of various neuropathologies.

Although neurotoxicity is a critical barrier to application of $\mathrm{Mn}^{2+}$ as a useful MRI contrast agent in clinical diagnosis, a chelated $\mathrm{Mn}^{2+}$ contrast agent, Mn-dipyridoxaldiphosphate (MnDPDP), is necessary to decrease the high acute toxicity of the free metal ions. It has been clinically approved for hepatic and pancreatic imaging for several years[19, 20], and also for assessments of myocardial function and viability in animal models[21]. This chelate gradually releases $\mathrm{Mn}^{2+}$ in presence of trans-metallation with zinc which is a Mn analog, allowing the detection of abnormalities by $\mathrm{Mn}^{2+}$ enhancement. MnDPDP has also been shown of certain virtue with antioxidative and cardioprotective properties[22]. In this study, we attempt to test the feasibility of delayed MnDPDP administration for detection of cerebral degeneration in $\mathrm{H}-\mathrm{I}$ rats. It may open the possibility of a future study of MnDPDP as a 'slow release' $\mathrm{Mn}^{2+}$ agent for clinical diagnosis of various neuropathologies.

\section{MATERIALS AND METHODS}

\section{Animal preparation}

All animal experiments were approved by the local animal research ethics committee. Pregnant Sprague-Dawley rats were obtained approximately 2 days before parturition, and their litters were culled to 9 to 13 pups. Neonate rats were kept with their mother in regular light/dark cycle for 7 days after birth (P7). The P7 rats, ranging from 12 to $16 \mathrm{~g}$ in weight, underwent unilateral ligation of right common carotid artery (double ligation followed by severing the artery in between) via a midline neck incision after anesthesia with $0.2 \mathrm{~mL}$ of inhalational isoflurane $[17,23-25]$. The surgery took approximately $5 \mathrm{~min}$ per pup, after which the pups were kept in an incubator for observation at $34^{\circ} \mathrm{C}$ for about $10-15 \mathrm{~min}$. On regaining normal movements, they were returned to their 
mother for nursing for $1 \mathrm{~h}$. These pups were subsequently placed in a hypoxic chamber of $8 \% \mathrm{O}_{2} / 92 \% \mathrm{~N}_{2}$ maintained at ambient temperatures $36^{\circ} \mathrm{C}$ for $1.5 \mathrm{~h}$. Among a total of $16 \mathrm{P} 7$ rats, 12 rats underwent H-I insult, among which four died during surgery or anoxia. Four rats were used as sham-operated controls; their left carotid artery was simply isolated and there was no exposure to hypoxia. The neonatal rats $(\mathrm{N}=12)$ were then divided into two groups. Group $1(\mathrm{~N}$ $=8$ ): H-I insult with intraperitoneal (IP) MnDPDP administration; Group $2(\mathrm{~N}=4)$ ): sham controls (without H-I insult) with IP MnDPDP administration. MnDPDP with $10 \mu \mathrm{mol} / \mathrm{mL}$ (Teslascan ${ }^{\circledR}$, Nycomed Imaging AS, Oslo, Norway) was injected at dosage of $0.1 \mu \mathrm{mol} / \mathrm{g}$ by intraperitoneal route in P14 while the rats were anesthetized with $1 \%$ isoflurane mixed with room air. This dosage is far lower than the human dosage $(5 \mu \mathrm{mol} / \mathrm{kg})$ according to converted method of body surface area calculation (equivalent dosage ratio between the human and P14 young rat is approximately 378). Among all rats undergoing MnDPDP administration, no abnormal behavior was observed after IP injection.

\section{MRI protocol}

All MRI experiments were performed on a 7-Tesla MRI scanner with maximum gradient of $360 \mathrm{mT} / \mathrm{m}(70 / 16$ PharmaScan, Bruker Biospin GmbH, Germany) using a 23 $\mathrm{mm}$ mouse brain coil (for rats of 3 weeks old or younger) and a $38 \mathrm{~mm}$ rat brain coil (for rats older than 3 weeks). During the MRI scan, the rats were positioned in a plastic holder and anesthetized by isoflurane $(1-1.5 \mathrm{vol} \%$ at $0.8-1 \mathrm{~L} / \mathrm{min}$ air flow via a nose cone) with respiratory monitoring. 2D $\mathrm{T}_{1}$-weighted images ( $\left.\mathrm{T}_{1} \mathrm{WIs}\right), \mathrm{T} 1 \mathrm{maps}, \mathrm{T}_{2}$-weighted images $\left(\mathrm{T}_{2} \mathrm{WIs}\right)$ and $\mathrm{T} 2$ maps were scanned in succession in P10, P14, P15, P21. Standard multislice coronal images were obtained with $\mathrm{FOV}=2.5 \mathrm{~cm}$, slice thickness $=0.5 \mathrm{~mm}$, matrix size $=256$ $\times 256$, voxel size $=98 \mu \mathrm{m} \times 98 \mu \mathrm{m} \times 500 \mu \mathrm{m}$, and 20 slices. T1WI were acquired by a fast spin echo (FSE) sequence with $\mathrm{TR}=500 \mathrm{~ms}, \mathrm{TE}_{\text {eff }}=7 \mathrm{~ms}$, echo train length $(\mathrm{ETL})=2$, number of averages $(\mathrm{NSA})=6$, and acquisition time $=5 \mathrm{~min}$. $\mathrm{T} 2 \mathrm{WI}$ were acquired using an FSE sequence with $\mathrm{TR}=6000$ $\mathrm{ms}, \mathrm{TE}_{\text {eff }}=60 \mathrm{~ms}, \mathrm{ETL}=12, \mathrm{NSA}=2$, and acquisition time $=3$ min. The T2 maps were acquired by a multislice and multiecho SE sequence, Bandwidth $=65789 \mathrm{~Hz}, \mathrm{TR}=3450 \mathrm{~ms}$, Echo effective $=10 \mathrm{~ms}$, Echo spacing $=10 \mathrm{~ms}$, Number of echo $=16, \mathrm{NSA}=1, \mathrm{Scan}$ time $=11 \mathrm{~min}$. The corresponding $\mathrm{T} 1$ maps were acquired by a saturation recovery method with a standard multislice spin-echo sequence, Bandwidth $=83333 \mathrm{~Hz}$, Five different TR values $=3200,1600$, 800,400 and $200 \mathrm{~ms}, \mathrm{TE}=7.2 \mathrm{~ms}, \mathrm{NSA}=1$, Spatial resolution $=98 \times 125 \mu \mathrm{m} /$ pixel, $\mathrm{FOV}=2.5 \times 2.5 \mathrm{~cm}$, slice thickness $=1 \mathrm{~mm}$, matrix $=200 \times 256$, scan time $=$ $15 \min 30 \mathrm{sec}$.

\section{Data analysis}

Images were analyzed using the public domain software ImageJ (National Institutes of Health [NIH], Washington, DC, USA) and ParaVision (Bruker Biospin GmbH, Germany). Region of interest (ROI) analysis of the T1 maps and T2 map was performed and defined in specific brain regions after comparing the coronal images with a rat brain atlas. Three brain regions were analyzed: hippocampus, thalamus, and cortex. Mean values were compared using two-tailed Student's t-tests, and the values along the time course were compared using ANOVA. Results were considered to be significantly different when $\mathrm{p}<0.05$. All statistical analyses were performed by using the statistical package SPSS for Windows (SPSS Inc., IL, USA). The results were presented as mean \pm SEM.

\section{RESULTS AND DISCUSSION}

\section{MEMRI in normal young rats by IP injection of MnDPDP}

After 1 day post-MnDPDP-injection, the contrast and signal enhancement were observed throughout the brain in T1WI images, suggesting $\mathrm{Mn}^{2+}$ release by MnDPDP was distributed throughout the brain. Higher contrast was observed in the periventricular regions, caudate-putamen, thalamus, the hippocampus, the olfactory bulb and cerebellum, which was consistent with a previous study regarding MEMRI in young rat brain during postnatal development[26]. In this study, the shortened percents of T1 value in cortex, thalamus and hippocampus were $10.4 \%$, $17.3 \%$ and $15.5 \%$ respectively in 1 day post-MnDPDP-injection. Such changes in T1 value were statistically significant (ANOVA, $p>0.05$ ) when compared to the same regions in the normal rats before MnDPDP injection (see Fig.1). However, these MnDPDP-induced changes were noticeably smaller than the reports in several previous studies [26, 27], which are related to the low dosage of manganese used in current study, and the lower absorption of MnDPDP in brain comparing with $\mathrm{MnCl}_{2}$ [28]. Moreover, $\mathrm{Mn}$ accumulation in brain from MnDPDP is constant for more than 2 weeks[28]. Thus, further decreases of T1 value in cortex, thalamus and hippocampus (shortened percents: $12.8 \%, 19.6 \%$ and $18.9 \%$ respectively) were found in 7 day post-MnDPDP-injection (see Fig.1), and that decreases of $\mathrm{T} 2$ value in cortex and thalamus were statistically significant (ANOVA, $\mathrm{p}>0.05$ ) only during 7 day postMnDPDP-injection when compared to the same regions in the normal rats before MnDPDP injection(see Fig.2).

\section{MEMRI detection of Neurodegeneration in H-I young rats by IP injection of MnDPDP}

All 8 neonatal rats induced with medium H-I injury exhibited lesions on the parietal cortex and striatum with distinct atrophy in the hemisphere ipsilateral to carotid artery 
ligation side (see Fig.3). At day 7 after H-I insult (P14), the lesion sites could not be detected in T1WIs before MnDPDP administration. But some lesions in subcortical white matter were presented as hyperintensity in T2WIs and T2 maps (the red arrows in Fig. 3), which was consistent with a previous study [29]. Moreover, some weak dark contrast also appeared in the ispilateral cortex (the yellow arrows in Fig. 3) and/or striatum in T2WIs and T2 maps, which is likely resulted from the endogenous iron accumulation due to an abnormal iron metabolism in $\mathrm{HI}$ injury[17]. Such decreases in T2 value were statistically significant $(t$ test, $\mathrm{p}>0.001)$ when compared to the same regions in the contralateral side (see Fig.4).

$\square$ Pre-MnDPDP(P14) $\square$ 1day post-MnDPDP(P15) 7days post-MnDPDP(P21)

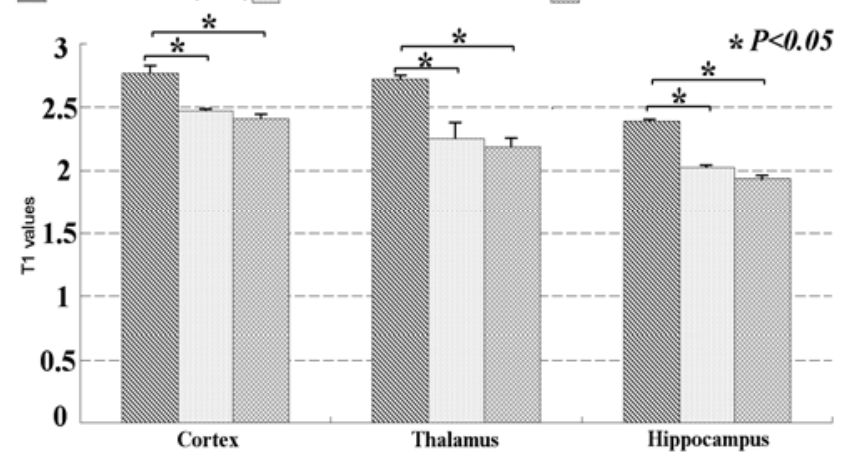

Fig. 1 The changes of $\mathrm{T} 1$ value in postnatal normal rat brains after IP injection of MnDPDP $(n=4)$.

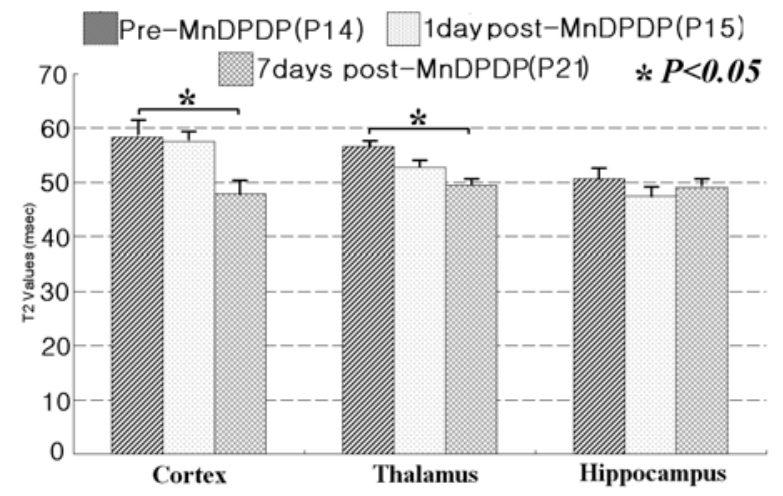

Fig. 2 The changes of $T 2$ value in postnatal normal rat brains after IP injection of MnDPDP $(n=4)$.

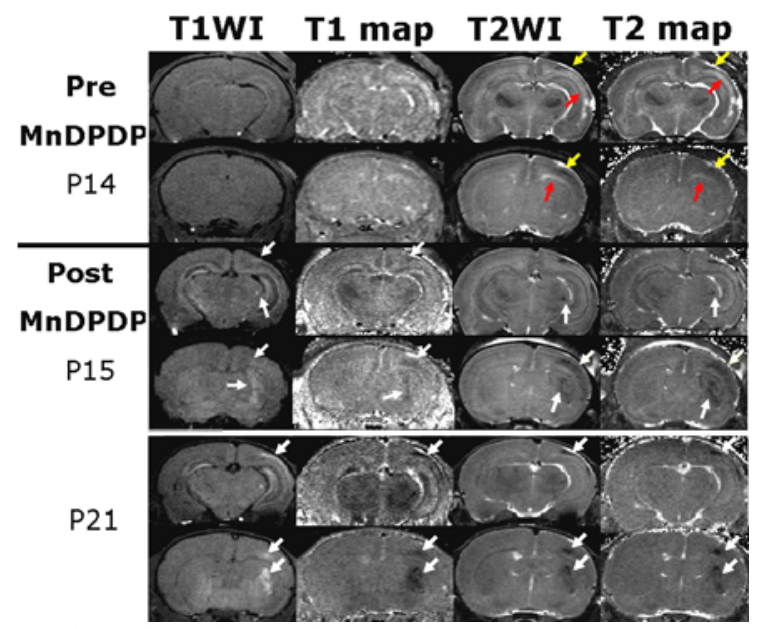

Fig. 3 Typical T1WIs, T1 maps, T2WIs and T2 maps in young rat brain of H-I injury before and after systemic MnDPDP administration.
At 1 day after MnDPDP injection (P15), some weak bright regions became visible in ispilateral cortex and striatum in T1WIs, suggesting MnDPDP induced enhancement, and that most spatially corresponded to regions with new dark contrast in T1 maps, T2WIs and T2 maps (the white arrows indicated in middle two rows of Fig. 3). Moreover, the decreases of $\mathrm{T} 1$ value in ipsilateral cortex and thalamus were statistically significant (ANOVA, $p>0.001$ ) when compared to the same regions prior to MnDPDP injection (see Fig.5). However, such decreases of T2 value were not statistically significant different in comparison with the data before MnDPDP injection (Fig.4). It suggests that MnDPDP induced change in T1 value is more significant than it is in $\mathrm{T} 2$ value.

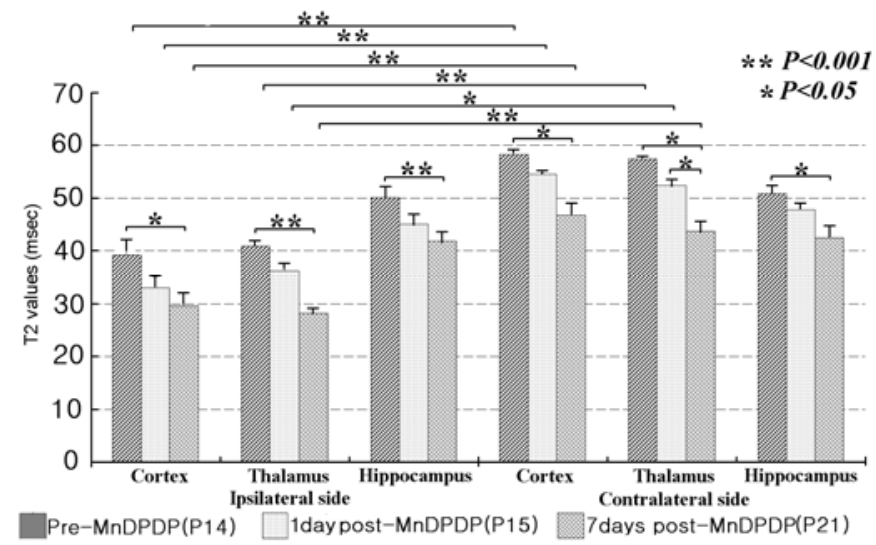

Fig. 4 The changes of $T 2$ value in $\mathrm{H}-\mathrm{I}$ rat brains after IP injection of $\operatorname{MnDPDP}(\mathbf{n}=\mathbf{8})$.

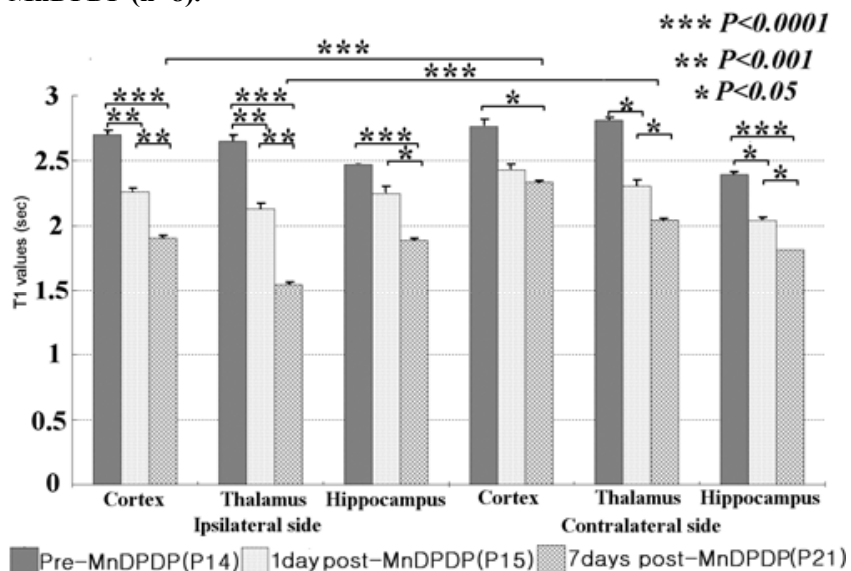

Fig. 5 The changes of T1 value in H-I rat brains after IP injection of $\operatorname{MnDPDP}(\mathrm{n}=8)$.

After 7 days post-MnDPDP-injection (P21), the $\mathrm{Mn}^{2+}$ induced contrast changes in ipsilateral cortex and striatum were further enhanced in T1WIs, T1 maps, T2WIs and T2 maps (the white arrows indicated in below two rows of Fig. 3). In Fig.5, the further decreases of T1 value in ipsilateral cortex and thalamus were statistically significant when compared to the same regions in 1 day after MnDPDP injection (ANOVA, $\mathrm{p}>0.001)$ and in the contralateral side $(t$ test, $\mathrm{p}>0.0001)$. The shortened percents of $\mathrm{T} 1$ value in lesion of ipsilateral cortex and thalamus were $29.6 \%$ and $41.7 \%$ respectively in 7 days 
post-MnDPDP-injection and the decreases in $\mathrm{T} 1$ value were statistically significant (ANOVA, $p>0.0001$ ) when compared to the same regions before MnDPDP injection (see Fig.5). Moreover, such decreases of T2 value were statistically significant different in comparison with the data before MnDPDP injection (see Fig.4). It suggests that Mn accumulation from MnDPDP in lesion sites is sustained and MEMRI for detection of H-I lesion sites in 7 days post-MnDPDP-injection is more pronounced than it is in 1 day post-MnDPDP-injection.

\section{CONCLUSION}

In this study, MnDPDP as a clinically approved manganese contrast medium for hepatic and pancreatic imaging was employed for the first time for MEMRI in both normal and H-I young rat brains. After a single intraperitoneal injection of low dose at $0.1 \mu \mathrm{mol} / \mathrm{g}$, Manganese enhancement induced by MnDPDP was observed as delayed pattern and more pronounced in 7 days post-injection. Moreover, such $\mathrm{Mn}$ accumulation from MnDPDP in H-I brains was pronounced in the H-I lesion sites and was detectable in T1WI, T1 map, T2WI and T2 map. The results demonstrated here support the possibility of using MnDPDP as a 'slow release' $\mathrm{Mn}^{2+}$ agent for clinical diagnosis of various neuropathologies in the future.

\section{REFERENCES}

[1]Y. J. Lin and A. P. Koretsky, "Manganese ion enhances T1-weighted MRI during brain activation: an approach to direct imaging of brain function," Magn Reson Med, vol. 38, pp. 378-88, Sep 1997.

[2]I. Aoki, S. Naruse, and C. Tanaka, "Manganese-enhanced magnetic resonance imaging (MEMRI) of brain activity and applications to early detection of brain ischemia," NMR Biomed, vol. 17, pp. 569-80, Dec 2004.

[3]Y. Z. Wadghiri, J. A. Blind, X. Duan, C. Moreno, X. Yu, A. L. Joyner, and D. H. Turnbull, "Manganese-enhanced magnetic resonance imaging (MEMRI) of mouse brain development," NMR Biomed, vol. 17, pp. 613-9, Dec 2004.

[4]I. Aoki, Y. J. Wu, A. C. Silva, R. M. Lynch, and A. P. Koretsky, "In vivo detection of neuroarchitecture in the rodent brain using manganese-enhanced MRI," Neuroimage, vol. 22, pp. 1046-59, Jul 2004.

[5]I. Aoki, T. Ebisu, C. Tanaka, K. Katsuta, A. Fujikawa, M. Umeda, M. Fukunaga, T. Takegami, E. M. Shapiro, and S. Naruse, "Detection of the anoxic depolarization of focal ischemia using manganese-enhanced MRI," Magn Reson Med, vol. 50, pp. 7-12, Jul 2003.

[6]R. G. Pautler, R. Mongeau, and R. E. Jacobs, "In vivo trans-synaptic tract tracing from the murine striatum and amygdala utilizing manganese enhanced MRI (MEMRI)," Magn Reson Med, vol. 50, pp. 33-9, Jul 2003.

[7]J. Crossgrove and W. Zheng, "Manganese toxicity upon overexposure," NMR Biomed, vol. 17, pp. 544-53, Dec 2004.

[8]A. Takeda, "Manganese action in brain function," Brain Res Brain Res Rev, vol. 41, pp. 79-87, Jan 2003.

[9] K. C. Chan, Q. L. Fu, E. S. Hui, K. F. So, and E. X. Wu, "Evaluation of the retina and optic nerve in a rat model of chronic glaucoma using in vivo manganese-enhanced magnetic resonance imaging," Neuroimage, vol. 40, pp. 1166-74, Apr 152008.

[10] K. C. Chan, Q. L. Fu, K. F. So, and E. X. Wu, "Evaluation of the visual system in a rat model of chronic glaucoma using manganese-enhanced magnetic resonance imaging," Conf Proc IEEE Eng Med Biol Soc, vol. 2007, pp. 67-70, 2007.

[11] M. Fujioka, T. Taoka, Y. Matsuo, K. Mishima, K. Ogoshi, Y. Kondo, M. Tsuda, M. Fujiwara, T. Asano, T. Sakaki, A. Miyasaki, D. Park, and B. K. Siesjo, "Magnetic resonance imaging shows delayed ischemic striatal neurodegeneration," Ann Neurol, vol. 54, pp. 732-47, Dec 2003.
[12] I. Suarez, G. Bodega, and B. Fernandez, "Glutamine synthetase in brain: effect of ammonia," Neurochem Int, vol. 41, pp. 123-42, Aug-Sep 2002.

[13] F. C. Wedler and R. B. Denman, "Glutamine synthetase: the major Mn(II) enzyme in mammalian brain," Curr Top Cell Regul, vol. 24, pp. 153-69, 1984.

[14] H. J. Bidmon, K. Kato, A. Schleicher, O. W. Witte, and K. Zilles, "Transient increase of manganese-superoxide dismutase in remote brain areas after focal photothrombotic cortical lesion," Stroke, vol. 29, pp. 203-10; discussion 211, Jan 1998.

[15] J. Lindenau, H. Noack, H. Possel, K. Asayama, and G. Wolf, "Cellular distribution of superoxide dismutases in the rat CNS," Glia, vol. 29, pp. 25-34, Jan 12000.

[16] K. C. Chan, K. X. Cai, H. X. Su, V. K. Hung, M. M. Cheung, C. T. Chiu, H. Guo, Y. Jian, S. K. Chung, W. T. Wu, and E. X. Wu, "Early detection of neurodegeneration in brain ischemia by manganese-enhanced MRI," Conf Proc IEEE Eng Med Biol Soc, vol. 2008, pp. 3884-7, 2008.

[17] J. Yang, P. L. Khong, Y. Wang, A. C. Chu, S. L. Ho, P. T. Cheung, and E. X. Wu, "Manganese-enhanced MRI detection of neurodegeneration in neonatal hypoxic-ischemic cerebral injury," Magn Reson Med, vol. 59, pp. 1329-39, Jun 2008.

[18] J. Yang and E. X. Wu, "Detection of cortical gray matter lesion in the late phase of mild hypoxic-ischemic injury by manganese-enhanced MRI," Neuroimage, vol. 39, pp. 669-79, Jan 152008.

[19] D. M. Koh, G. Brown, Z. Meer, A. R. Norman, and J. E. Husband, "Diagnostic accuracy of rim and segmental MRI enhancement of colorectal hepatic metastasis after administration of mangafodipir trisodium," AJR Am J Roentgenol, vol. 188, pp. W154-61, Feb 2007.

[20] D. Sahani, S. R. Prasad, M. Maher, A. L. Warshaw, P. F. Hahn, and S. Saini, "Functioning acinar cell pancreatic carcinoma: diagnosis on mangafodipir trisodium (Mn-DPDP)-enhanced MRI," $J$ Comput Assist Tomogr, vol. 26, pp. 126-8, Jan-Feb 2002.

[21] H. Brurok, T. Skoglund, K. Berg, S. Skarra, J. O. Karlsson, and P. Jynge, "Myocardial manganese elevation and proton relaxivity enhancement with manganese dipyridoxyl diphosphate. Ex vivo assessments in normally perfused and ischemic guinea pig hearts," NMR Biomed, vol. 12, pp. 364-72, Oct 1999.

[22] H. Brurok, J. H. Ardenkjaer-Larsen, G. Hansson, S. Skarra, K. Berg, J. O. Karlsson, I. Laursen, and P. Jynge, "Manganese dipyridoxyl diphosphate: MRI contrast agent with antioxidative and cardioprotective properties?," Biochem Biophys Res Commun, vol. 254, pp. 768-72, Jan 271999.

[23] Y. Wang, P. T. Cheung, G. X. Shen, E. X. Wu, G. Cao, I. Bart, W. H. Wong, and P. L. Khong, "Hypoxic-ischemic brain injury in the neonatal rat model: relationship between lesion size at early MR imaging and irreversible infarction," AJNR Am J Neuroradiol, vol. 27, pp. 51-4, Jan 2006.

[24] Y. Wang, P. T. Cheung, G. X. Shen, I. Bhatia, E. X. Wu, D. Qiu, and P. L. Khong, "Comparing diffusion-weighted and T2-weighted MR imaging for the quantification of infarct size in a neonatal rat hypoxic-ischemic model at 24h post-injury," Int J Dev Neurosci, vol. 25, pp. 1-5, Feb 2007.

[25] S. Wang, E. X. Wu, C. N. Tam, H. F. Lau, P. T. Cheung, and P. L. Khong, "Characterization of white matter injury in a hypoxic-ischemic neonatal rat model by diffusion tensor MRI," Stroke, vol. 39, pp. 2348-53, Aug 2008.

[26] P. L. de Sousa, S. L. de Souza, A. C. Silva, R. E. de Souza, and R. M. de Castro, "Manganese-enhanced magnetic resonance imaging (MEMRI) of rat brain after systemic administration of $\mathrm{MnCl} 2$ : changes in $\mathrm{T} 1$ relaxation times during postnatal development," J Magn Reson Imaging, vol. 25, pp. 32-8, Jan 2007.

[27] J. H. Lee, A. C. Silva, H. Merkle, and A. P. Koretsky, "Manganese-enhanced magnetic resonance imaging of mouse brain after systemic administration of $\mathrm{MnCl} 2$ : dose-dependent and temporal evolution of T1 contrast," Magn Reson Med, vol. 53, pp. 640-8, Mar 2005.

[28] B. Gallez, C. Baudelet, and M. Geurts, "Regional distribution of manganese found in the brain after injection of a single dose of manganese-based contrast agents," Magn Reson Imaging, vol. 16, pp. 1211-5, Dec 1998.

[29] M. Qiao, S. Meng, K. Scobie, T. Foniok, and U. I. Tuor, "Magnetic resonance imaging of differential gray versus white matter injury following a mild or moderate hypoxic-ischemic insult in neonatal rats," Neurosci Lett, vol. 368, pp. 332-6, Sep 302004. 